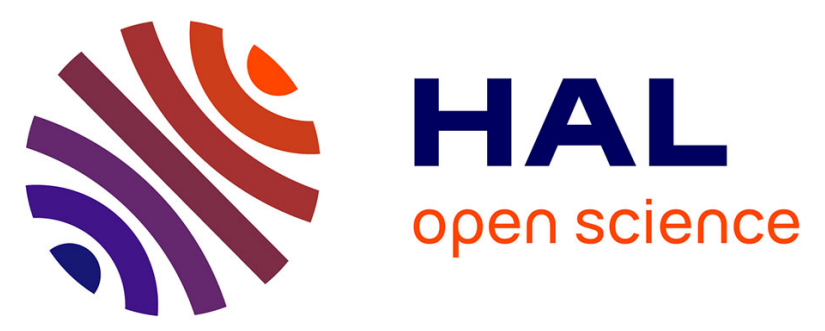

\title{
Etude du processus de catégorisation chez des patientes aux troubles des conduites alimentaires: une nouvelle approche cognitive de la psychopathologie
}

Isabel Urdapilleta, C Mirabel-Sarron, Jean-Marc Meunier, J.-F Richard

\section{- To cite this version:}

Isabel Urdapilleta, C Mirabel-Sarron, Jean-Marc Meunier, J.-F Richard. Etude du processus de catégorisation chez des patientes aux troubles des conduites alimentaires: une nouvelle approche cognitive de la psychopathologie. L'Encéphale, 2005, 31 (1), pp.82 - 91. 10.1016/S0013-7006(05)82376-0 . hal01503772

HAL Id: hal-01503772

https://hal-univ-paris8.archives-ouvertes.fr/hal-01503772

Submitted on 7 Apr 2017

HAL is a multi-disciplinary open access archive for the deposit and dissemination of scientific research documents, whether they are published or not. The documents may come from teaching and research institutions in France or abroad, or from public or private research centers.
L'archive ouverte pluridisciplinaire HAL, est destinée au dépôt et à la diffusion de documents scientifiques de niveau recherche, publiés ou non, émanant des établissements d'enseignement et de recherche français ou étrangers, des laboratoires publics ou privés. 
Etude du processus de catégorisation chez des patientes aux troubles des conduites alimentaires : une nouvelle approche cognitive de la psychopathologie

\author{
I. URDAPILLETA ${ }^{1}$, C. MIRABEL-SARRON ${ }^{2}$, J.-M. MEUNIER ${ }^{1}$, J.-F. RICHARD ${ }^{1}$
}

1 Laboratoire CNRS FRE 2048, Cognition et Activités Mentales Finalisées, Université de Paris VIII, 2 rue de la Liberté 93526 Saint Denis - France.

2 Equipe de recherche en psychologie clinique. Université Paris VIII 2 rue de la Liberté - 93526 Saint-Denis - France.

Clinique des Maladies Mentales et de l'Encéphale, Hôpital Sainte Anne 100, rue de la Santé, 75014 Paris - France. 


\section{Study of the categorization process among patients with eating disorders: a new cognitive approach to psychopathology}

\section{Abstract}

Anorexic and bulimic patients have a highly distorted relationship with food and eating, even though they tend to be knowledgeable about diet and nutrition. The progress of this disease, as well as its complications and associated difficulties, are increasingly understood, while the etiopathogeny of eating disorders remains obscure. The approach that we are proposing involves the study of one of the most fundamental cognitive functions of human reasoning - the cognitive process of categorization. The purpose of this study is to understand the procedures used by these patients to construct representations of food.

Categorization, one of the basic features of human cognition, allows individuals to organize their subjective experience of the surrounding environment by structuring its contents. This ability to group different objects into the same category based on their common characteristics is important for explaining the major cognitive activities of planning, memorization, communication and perception. Indeed, our categories reflect our conceptions of the world. They depend on our experiences and representations, as well as the expertise acquired in a specific field. The differences that appear in the categories created by subjects when they are asked to classify objects reveal the properties that are most salient to them and, as a result, the interests, values and ideas associated with these properties. There are three types of properties: perceptive properties, which describe the object's shape, color, odor and texture; structural properties, which relate to the object's components; and functional properties, which specify the way in which the object is used and provide an answer to the question, "What is it used for?" Subjects attribute these functional properties by means of knowledge or inference according to their representation of the object's role; such properties are especially likely to emerge during top-down (theory-driven) processing. The type of processing used (bottom-up or top-down) is dependent on a certain number of factors. We hypothesize, within the context of food product categorization, that patients suffering from eating disorders largely resort to processing based on acquired information or beliefs about the objects, i.e. top-down processing.

We present two studies: a naturalistic and exploratory pilot study whose goal is to identify whether the various categorization processes used by eating disorder patients differ from those employed by subjects not suffering from an eating disorder. A second study aims to identify the different categorization procedures.

During the first experiment, 68 women (17 control subjects, 17 anorexics, 17 anorexic bulimics and 17 bulimics) aged 18-39 (average age: 26.6) verbalize all representations that come to mind during 
a limited time period as the name of a food item is read. Eighty-nine food items are presented in alphabetical order. The list is read out loud and all comments are recorded.

The data is processed in tree ways: an analysis based on the positive or negative valence of each representation, an analysis based on each categories of food and an analysis of representations based on themes expressed. The tree analyses (valence, categories of food and theme assigned to the representations) show differences between the representations of the four experimental groups. In fact, the anorexics and anorexic bulimics mainly express strongly negatives representations about food, whereas bulimics and control produce representations whose positive and negative valences balances. These negative cognitions concern mainly meat for the control subjects and cakes for the subjects reached of TCA. Concerning theme assigned to the representations, the control subjects produce mainly cognitions relating to the hedonism, the flavor of food and their purpose on health. The anorexics and anorexics-bulimics evoke mainly the fat and sugar content of the foods. The bulimics evoke mainly cognitions relating to the effect on health and the intestinal transit time of food. These results lead one to believe that it is not the bulimic binging and purging of these patients, but rather their restrictive behavior that is the determining factor in the differences in food representations observed between the two experimental groups.

During the second experiment, 60 women (15 controls, 15 anorexics, 15 anorexic bulimics and 15 bulimics) aged 18-32 (average age: 25.6) classified 27 food names according to their similarities and differences, and then explained the reasons for their categorizations. The data were analyzed in terms of similarity/difference, and the verbalizations were analyzed by content.

The results indicate that 10 of the 27 foods were categorized differently by the controls and the subjects with eating disorders. Subjects classified the following foods: camembert cheese, $\underline{\text { cold cuts, }}$

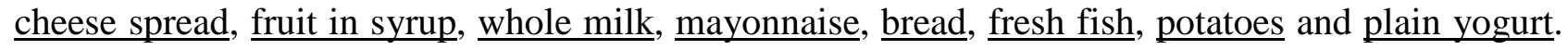
Bulimics and controls use similar classifications for food names, while anorexics and atypical bulimics classify foods in a similar way.

Examining the categorization criteria used during verbalizations allows us to better understand these differences. The control group's major criterion seems to be the succession of dishes. These subjects group into separate categories entry foods (beef, eggs, fish, etc.), vegetables, cheese or $\underline{\text { dairy foods, }}$ and finally desserts. Additional foods, like bread and mayonnaise, belong to the same category. Other categories are nutritional criteria (for example, dairy products contain calcium) and biological criteria (for example, bananas and apples are fruits). These categorization criteria include structural properties (which describe what the object is made of) and functional, "academic" properties, those which describe how foods are used, "as in cookbooks or diet books." On the other hand, the categorization criteria expressed by anorexic patients are very different from those used by control subjects: foods that are hard to eliminate, rich, high-fat and therefore indigestible are 


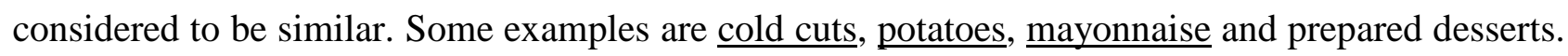
A second categorization criterion involves the concept of natural foods: certain foods "are unhealthy because they're processed, so they're bad for you" - one such example is cheese spread. A third criterion concerns the notion of familiar foods: poultry and eggs, for example, are "familiar to us." We are clearly seeing here the importance of functional properties in the categorization of

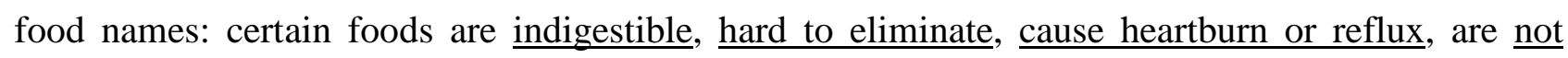
$\underline{\text { natural, }}$ and thus are avoided. The categorization criteria mentioned by bulimic patients also clearly take into account the functional properties of foods. The criteria are of the following type: "it's filling, it relieves a bulimic attack, it helps prevent heartburn and constipation, etc." It appears that bulimics' categorization criteria are solely associated with these foods' imagined or real effect on the body. The categorization criteria used by anorexic bulimics seem to be especially associated with weight gain or the consumption of such foods during bulimia attacks because "they make you feel full." On the other hand, light foods, which patients allow themselves to eat, are placed in the same category.

This study, which seeks to understand the cognitive functioning of eating disorder patients with anorexia and bulimia, has brought new elements to light. All patients exhibit food categorization processes that differ greatly from those displayed by control subjects. Patients also attribute greater significance to the functional properties of foods as compared to controls, who give priority to structural properties. Anorexic and bulimic patients base their food categorizations on the consequences of ingestion, in terms of health, digestion and weight gain. Their processing of food stimuli is therefore radically different and gives a dominating place to top-down processes. Additionnal studies should supplement these findings in order to gain a better understanding of patients' disturbed processing of information.

Key words: anorexia - bulimia - cognition - categorization - property

Mots clés : anorexie - boulimie - cognition - catégorisation - propriété 


\section{Introduction}

Les troubles des conduites alimentaires constituent une pathologie fréquente qui touche principalement les jeunes filles dès l'âge de la puberté. Ils sont considérés aujourd'hui comme un véritable problème de santé publique. En effet, l'évolution de cette pathologie alimentaire est grevée de nombreuses complications pouvant mettre en jeu le pronostic vital. L'anorexie est un trouble mental qui a un taux de mortalité de $6 \%$ par décennie d'évolution dans la maladie (20), et il n'existe actuellement aucun traitement pharmacologique de l'anorexie. Ces troubles des conduites alimentaires se présentent comme des troubles souvent chroniques, pour lesquels les différentes propositions thérapeutiques s'avèrent décevantes que ce soit pour les troubles restrictifs, anorexiques, ou pour les troubles boulimiques.

Ainsi, dans une optique de prévention, il est important de tenter de mieux comprendre cette pathologie qui nécessite la mise en œuvre de traitements médicaux et psychologiques spécialisés. Pourtant si l'évolution de cette maladie, les complications, les troubles associés sont de mieux en mieux connus, en revanche l'étiopathogénie des troubles des conduites alimentaires reste obscure. Différentes recherches ont tenté de mieux cerner les éléments cliniques, les désordres biologiques, physiologiques et hormonaux, ceux touchant le fonctionnement métabolique, les facteurs chimiques cérébraux, ainsi que les différentes composantes psychologiques intervenant dans ces troubles (36). Ces recherches n'ont pas toujours été couronnées de succès. L'approche que nous proposons concerne l'exploration des fonctions cognitives de patientes atteintes d'un trouble des conduites alimentaires.

Deux types d'observations motivent ces explorations. Les premières sont d'ordre clinique. En effet, tout thérapeute est frappé par le type de discours exprimé par ces patientes : la perception qu'elles ont de l'environnement, du corps, des aliments est étonnante, comme le souligne Bruch (5). Les secondes observations proviennent de spécialistes des cognitions qui décrivent des fonctionnements cognitifs propres aux sujets atteints de troubles des conduites alimentaires. Certains auteurs (19; 16 ; 13), adoptent une approche descriptive et concluent qu'un des facteurs de maintien et de chronicisation des troubles est lié aux représentations particulières des aliments, de leurs conséquences sur le corps, et du partage des aliments avec autrui. D'autres auteurs $(15 ; 3 ; 2 ; 8$; 12) qui optent pour une démarche plus expérimentale, montrent que le fonctionnement cognitif de ces patientes est perturbé. En revanche, il existe très peu de questionnaires ou échelles qui explorent cette dimension $(9 ; 18 ; 30 ; 38 ; 37 ; 29 ; 33 ; 14)$. Ainsi seul est traduit et validé en français l'Eating Disorder Inventory dans ses deux versions, qui permet l'exploration de huit dimensions cognitives telles que l'insatisfaction corporelle, le rapport aux autres, etc.

Des psychologues expérimentaux ont montré que le traitement de l'information serait modifié chez les patientes présentant un trouble des conduites alimentaires mais de manière différente en ce qui 
concerne les anorexiques et les boulimiques. Les conflits cognitifs porteraient bien davantage sur les aliments pour les anorexiques, alors qu'ils porteraient essentiellement sur l'appréhension du corps pour les boulimiques (9). Ce constat de dysfonctionnement cognitif mesuré cliniquement et expérimentalement par différentes investigations $(29 ; 28 ; 26)$ ne nous apporte pas d'élément de compréhension sur les étapes du traitement de l'information qui seraient altérées. L'étude de ces éléments pourraient par la suite nous apporter des données utilisables dans des programmes d'actions thérapeutiques.

Aussi, nous nous proposons d'étudier une des fonctions cognitives les plus essentielles du raisonnement humain, le processus cognitif de catégorisation, afin de connaître les procédures qu'utilisent ces patientes pour se construire leur représentation du monde environnant. Dans un premier temps, une étude pilote, naturaliste, exploratoire a pour but d'identifier si ces processus de catégorisation sont différents chez les patientes sujettes à des troubles alimentaires et chez des sujets ne présentant pas de trouble ; une deuxième étude plus approfondie a pour fonction d'identifier les dimensions sous-jacentes à ces processus de catégorisation.

\section{Les processus de catégorisation}

La catégorisation, l'une des caractéristiques essentielles de la cognition humaine, permet aux individus de découper l'environnement selon des classifications permettant de traiter de manière équivalente des stimulus non identiques. Elle permet ainsi à l'individu d'organiser son expérience subjective de l'environnement en structurant son contenu. Cette capacité à grouper des individus différents dans une même catégorie en fonction de leurs caractéristiques communes est importante non seulement pour expliquer le fonctionnement de la mémoire sémantique $(34 ; 39 ; 40)$, le développement cognitif $(6 ; 32)$, mais aussi les grandes activités cognitives que sont la planification de l'action, la mémorisation, la perception et la communication $(25 ; 41)$. Selon ces auteurs, nos catégories reflètent nos conceptions du monde. Elles dépendent à la fois de nos expériences et de nos représentations, des représentations culturelles, mais aussi de l'expertise acquise dans un domaine. Ainsi de nombreuses recherches mettent l'accent sur les différences culturelles et sur une variabilité interindividuelle de la catégorisation $(24 ; 43)$. Cela souligne l'influence indéniable du groupe sur la construction de la structure psychologique de l'individu. De la sorte, les différences qui apparaissent dans les regroupements opérés par les sujets quand on leur demande de classer des objets sont révélateurs des propriétés qui sont les plus saillantes pour eux et donc des intérêts, des valeurs et des conceptions attachées à ces propriétés. L’importance des propriétés dans l'activité de catégorisation des objets est admise dans toutes les théories de la catégorisation. On peut distinguer trois types de propriétés (11) : les propriétés perceptives, qui décrivent la forme de l'objet, sa couleur, son odeur ou la qualité de son toucher, par exemple: "La pomme est rouge, brillante, 


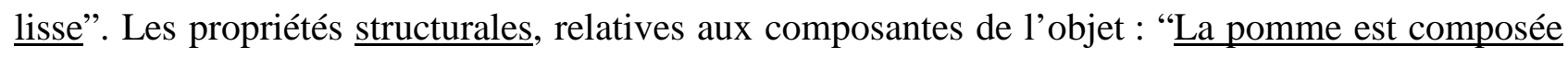
d'une peau, de chair". Les propriétés perceptives et structurales sont des propriétés visibles et mesurables (il existe des instruments pour en déterminer la taille, la longueur d'onde, la couleur, etc.). Ces propriétés sont de nature physique et peuvent être opposées à des propriétés de nature cognitive : les propriétés fonctionnelles qui précisent l'usage qui est fait de l'objet et permettent de répondre à la question : “A quoi cela sert-il ?" ; "La pomme est un aliment, elle nourrit ". Ces propriétés fonctionnelles sont attribuées par connaissance ou inférence en fonction de la représentation de l'usage que l'on a l'objet ; elles interviennent surtout lors des traitements descendants, guidés par les théories. Dans ces traitements, les représentations et croyances ont un rôle prépondérant (Bruner \& Postman, $1949 ; 21 ; 31 ; 42 ; 44 ; 45$ ).

De nombreux auteurs ont souligné des cognitions alimentaires propres aux sujets souffrant de troubles des conduites alimentaires $(7 ; 10 ; 22 ; 26$; Waller, Ohanian, Meyer \& Osman, 2000), cognition ayant un rôle important dans la régulation des comportements alimentaires (4). Aussi, nous faisons l'hypothèse, que dans une tâche de catégorisation de produits alimentaires, des patientes atteints de troubles des conduites alimentaires mettent en jeu de façon prédominante des traitements basés sur des représentations ou croyances acquises concernant les objets, c'est-à-dire des traitements descendants. Ainsi, l'activité de catégorisation étant très sensible aux théories sousjacentes des individus, nous l'utilisons dans un paradigme expérimental de regroupement en classes pour appréhender les représentations alimentaires de jeunes filles souffrant de troubles des conduites alimentaires

\section{Etudes expérimentales}

Deux études vont s'échelonner sur plusieurs années afin d'explorer les modalités de catégorisation de patientes aux troubles des conduites alimentaires.

\subsection{Etude pilote naturaliste}

Compte tenu de l'absence de données sur les processus de catégorisation de patientes anorexiques et boulimiques, nous avons été amenés à pratiquer une étude exploratoire afin d'estimer si notre hypothèse générale d'une catégorisation différente entre patientes et témoins était valide, ou si notre travail d'investigation devait s'arrêter là et être reformulé.

\subsubsection{Populations étudiées}

Soixante huit femmes ont passé cette expérimentation, soit 17 témoins (étudiantes en psychologie), 17 anorexiques, 17 anorexiques-boulimiques et 17 boulimiques (Cf. tableau 1). La classification des patientes dans un de ces quatre groupes a été réalisée en utilisant les critères diagnostiques de 
l'Organisation Mondiale de la Santé (ICD10). Les patientes ont entre 18 et 39 ans (âge moyen 26,6 ans). Pour chaque témoin, l'absence de trouble des conduites alimentaires est vérifiée par la passation d'un questionnaire (Eating Attitude Test 40 - Garner \& Garfinkel, 1979).

Tableau I - Effectif, âge moyen, BMI et score à l'E.A.T. des participantes

\begin{tabular}{|c|c|c|c|c|}
\hline Participantes & $\underline{\text { Effectif }}$ & Age moyen & B.M.I. & E.A.T. \\
\hline Témoins & 17 & 26,4 & $20,6 \pm 1,6$ & 20 \\
\hline Anorexiques & 17 & 26.6 & $14,7 \pm 1,5$ & XXXX \\
\hline Anorexiques-boulimiques & 17 & 26.5 & $16,2 \pm 1,6$ & XXXX \\
\hline Boulimiques & 17 & 26.8 & $22,1 \pm 1,5$ & XXXX \\
\hline
\end{tabular}

\subsubsection{Démarche expérimentale}

Elle consiste à faire associer en temps limité, à la lecture d'un item alimentaire toutes les représentations qui passent par l'esprit du sujet. Quatre vingt neuf items alimentaires sont proposés par ordre alphabétique par le même expérimentateur. Ils se répartissent en 7 catégories d'aliments : féculents, fromages, fruits, gâteaux, légumes, produits laitiers et viande. La liste est lue à voix haute et les verbalisations de chaque sujet sont entièrement notées puis codées par deux expérimentateurs. Par exemple, les cognitions recueillies sont des phrases du type "le chocolat est trop calorique" ou encore des images mentales de type " je vois ma grand-mère dans le jardin ".

Le traitement des données est effectué selon trois axes : une analyse selon la valence positive (c'est très bon, c'est jolie, j'aime, etc.) ou négative de chacune des représentations (c'est trop gras, c'est mauvais, etc.), une analyse selon les catégories d'aliments concernés par les représentations négatives et une analyse selon les types de cognition évoqués.

\subsubsection{Résultats}

Concernant la valence des représentations. Sur les 3582 propriétés verbalisées, 46,15\% d'entre elles (1653) sont à valence positive, 53,85\% (1929) sont à valence négatives. Toutefois les résultats font apparaître que la valence accordée aux représentations n'est pas la même selon les groupes ( $\mathrm{X}^{2}=111,30 ; \mathrm{p}<.001$ ). La comparaison des proportions de valence positive et de valence négative dans chaque groupe montre que les anorexiques $\left(\mathrm{X}^{2}\right.$ corr $\left.=42,23 ; \mathrm{p}<.001\right)$ et anorexiques-boulimiques $\left(\mathrm{X}_{\text {corr }}^{2}=63,87 ; \mathrm{p}<.001\right)$ manifestent plutôt des représentations à partir des aliments négatives. En revanche les témoins produisent davantage de cognitions positives $\left(X^{2}\right.$ corr $\left.=19,47 ; p<.001\right)$ tandis que les boulimiques n'expriment pas plus de représentations à valence positive que de représentations à valence négative $\left(\mathrm{X}_{\text {corr }}^{2}=4,88 ; \mathrm{ns}\right)$ 
Tableau II. - Répartition des cognitions selon leur valence positive ou négative pour chaque groupe de participantes.

\begin{tabular}{|c|c|c|c|c|c|c|}
\hline Participantes & Cogni & positives & $\underline{\text { Cogni }}$ & négatives & $\underline{\text { Total }}$ & \\
\hline Témoins & 342 & $59,3 \% *$ & 235 & $40,7 \%$ & 577 & $16,10 \%$ \\
\hline Anorexiques & 320 & $38,6 \%$ & 508 & $\underline{61,4 \% *}$ & 828 & $23,12 \%$ \\
\hline Anorexiques-boulimiques & 424 & $38,0 \%$ & 692 & $\underline{62,0 \% *}$ & 1116 & $31,16 \%$ \\
\hline Boulimiques & 567 & $53,4 \%$ & 494 & $\underline{46,6 \%}$ & 1061 & $29,62 \%$ \\
\hline Total & 1653 & $46,15 \%$ & 1929 & $53,85 \%$ & 3582 & $100 \%$ \\
\hline
\end{tabular}

* Comparaison entre cognitions positives et négatives significatives à $\mathrm{p}<.001$

Concernant les catégories d'aliments. Les cognitions négatives se répartissent sur les 7 catégories d'aliments de façon différentes en fonction des groupes expérimentaux. Ainsi comme le montre le tableau 3, ces cognitions négatives concernent pour les témoins principalement la viande $(23,7 \%$ des cognitions négatives), les gâteaux pour les anorexiques (18,3\%), anorexiques-boulimiques (17,5 $\%)$ et boulimiques $(18,9 \%)$. $\left(\mathrm{X}^{2}=34,26 ; \mathrm{p}<.01\right)$

Tableau III. - Répartition des cognitions négatives selon les groupes d'aliments pour chaque groupe de participantes.

\begin{tabular}{llllllll}
\hline Participantes & \multicolumn{2}{l}{ Féculentsfromages fruits } & gâteaux & légumes & produits laitiers & viande \\
\hline Témoins & $10,8 \%$ & $10,8 \%$ & 12,8 & $13,9 \%$ & $13,5 \%$ & $14,5 \%$ & $\underline{23,7 \%}$ \\
Anorexiques & $12,8 \%$ & $17,7 \%$ & $11,5 \%$ & $\underline{18,3 \%}$ & $11,5 \%$ & $12,7 \%$ & $15,5 \%$ \\
Ano.Boulimiques & $15,8 \%$ & $14,7 \%$ & $14,1 \%$ & $\underline{17,5 \%}$ & $11,5 \%$ & $14,7 \%$ & $11,7 \%$ \\
Boulimiques & $14,1 \%$ & $15,9 \%$ & $13,4 \%$ & $\underline{18,9 \%}$ & $11,8 \%$ & $11,7 \%$ & $14,2 \%$ \\
\hline
\end{tabular}

Concernant les thèmes abordés. Les verbalisations ont été codées en fonction de 12 thèmes :

Utilité pour le corps ( les gâteaux, cela n’apporte rien de positif au corps »).

Lien avec la crise de boulimie ( « le chocolat, j’en consomme en crise »).

Effet sur la digestion ( « la viande rouge c'est difficile à éliminer »).

Flaveur («la pomme a un goût acide »).

Teneur en gras ( $«$ le beurre, c'est une montagne de graisse »).

Hédonisme (j'aime / je n'aime pas).

Conséquences intestinales ( les légumes verts, cela permet de lutter contre la constipation »).

Caractère naturel ( le riz, c'est un produit naturel »).

Effet sur le poids ( « le sucre va directement dans mes cuisses »).

Effet sur la santé ( « les fruits, c'est bon pour la santé »).

Teneur en sucre («la confiture, c'est que du sucre »). 


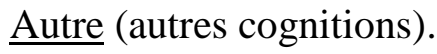

L'analyse de ces cognitions montre que (Cf. figure 1):

- Les témoins produisent principalement des cognitions relatives à l'hédonisme (non appréciation des aliments), à l'effet sur la santé des aliments («le beurre cuit n'est pas bon pour la santé ») et enfin à la flaveur des aliments (goût sucré, amer, etc.).

- Les anorexiques et anorexiques-boulimiques évoquent principalement la teneur en gras et en sucre des aliments. Les anorexiques évoquent aussi leur caractère naturel ou fabriqué et leurs effets sur la

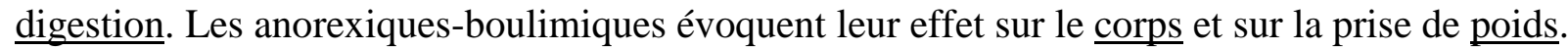

- Les boulimiques évoquent principalement des cognitions relatives à l'effet sur la santé et sur le transit intestinal des aliments. Des cognitions liées aux aliments consommés lors de crise ou encore à l'hédonisme sont aussi évoquées.

Insérer figure 1 par ici

Ces résultats laissent penser que ce ne sont pas les accès boulimiques, mais le comportement restrictif de ces patientes qui serait le facteur déterminant des différences de représentations alimentaires observées entre nos groupes expérimentaux.

\subsection{Deuxième temps expérimental}

Nous avons voulu compléter cette première étude exploratoire par un protocole expérimental systématisé ayant fait ses preuves dans d'autres domaines pour mettre en evidence des représentations alimentaires (35). Ce protocole utilise une tâche dans laquelle les sujets doivent classer des aliments (tâche de catégorisation) en fonction de leurs similarités et différences. Nous pensons ainsi mettre en évidence les représentations de ce qu'évoquent pour eux ces aliments afin de compléter les résultats issus de l'étude exploratoire.

\subsubsection{Populations étudiées}

Soixante femmes (qui n'ont pas été sujets de l'étude exploratoire) ont passé cette expérimentation, soit 15 témoins, 15 anorexiques, 15 anorexiques-boulimiques et 15 boulimiques (Cf. Tableau 4). Les patientes ont entre 18 et 32 ans (age moyen 25,6 ans). Les critères d'inclusion sont les mêmes que ceux de l'étude pilote naturaliste. 
Tableau IV. Effectif, âge moyen, BMI et score à l'E.A.T. des participantes

\begin{tabular}{lllll}
\hline Participantes & Effectif & Age moyen & B.M.I. & E.A.T. \\
\hline Témoins & 15 & 25.7 & $22,2 \pm 1,6$ & $8 \pm 8,5$ \\
Anorexiques & 15 & 25.6 & $14,5 \pm 1,5$ & XXXX \\
Anorexiques-boulimiques & 15 & 25.9 & $15,8 \pm 1,6$ & XXXX \\
Boulimiques & 15 & 25.1 & $21,1 \pm 1,5$ & $49 \pm 10,5$ \\
\hline
\end{tabular}

\subsubsection{Démarche expérimentale}

La tâche proposée aux sujets a consisté à classer 27 noms d'aliments en fonction de leurs similarités et de leurs différences, puis à exprimer les raisons pour lesquelles ils ont effectué ces regroupements. Ces 27 noms d'aliments ont été sélectionnés parmi les 89 aliments de l'étude exploratoire naturaliste afin de représenter les grandes catégories d'aliments distinguées en nutrition. Ce sont des produits de consommation courante pour toute personne de culture française. Ils ont été choisis en accord avec la diététicienne de la Clinique des Maladies Mentales et de l'Encéphale, hôpital Sainte-Anne. Ces aliments sont les suivants : banane, betterave, bœuf,

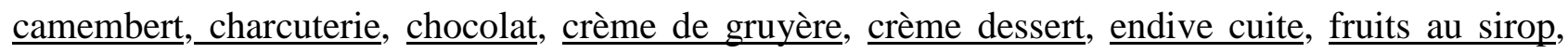
gâteau à la crème, gâteau sec, jus de fruit, lait entier, macédoine de légumes, mayonnaise, œuf, pain, petits pois, poire, poisson frais, pomme, pomme de terre, tarte aux fruits, $\underline{\text { tomate, }} \underline{\text { volaille, yaourt }}$ nature.

La consigne est la suivante : “27 noms d'aliments vont vous être proposés ; votre tâche consiste à classer les aliments qui vont ensemble". Puis "pouvez-vous décrire chacun des tas, en quoi ces aliments sont-ils semblables?".

\subsubsection{Analyse des Données}

Les données issues de la tâche de catégorisation ont été traitées par une analyse de proximité ; les verbalisations ont fait l'objet d'une analyse de contenu.

Concernant la tâche de catégorisation : Les participantes ont regroupé en un certain nombre de tas les 27 noms d'aliments en les classant les uns après les autres. Le nombre de tas étant différent selon les participantes, les résultats ont été traités par la méthode des scores (1). Cette méthode consiste dans un premier temps à calculer la fréquence d'association de tous les couples d'objets (ou aliments), c'est-à-dire le nombre de fois où deux objets sont rangés dans la même classe. On prend cette fréquence d'association comme mesure de proximité entre les objets. On considère alors que plus une paire d'objets apparaît fréquemment dans les protocoles, plus l'association entre les deux objets est stable. L'indice de similarité obtenu résulte du nombre de fois où deux objets sont 
classés dans une catégorie et forment une paire. On construit alors un tableau de similarité de "x" colonnes sur "x" lignes ("x" étant le nombre d'objets, soit ici 27) et pour chaque case du tableau, on indique la fréquence de classement de cette paire dans les classes. L'indice de similarité a ainsi une valeur minimale de 0 (aucun sujet n'a associé les deux objets du couple dans une même catégorie) et sa valeur maximale est de $\mathrm{N}$ (tous les sujets ont mis les deux objets du couple dans la même classe).

A partir de cette matrice, nous avons réalisé une analyse des proximités (avec le logiciel Statistica $\odot$ StatSoft, Inc) qui vise à présenter les objets dans un espace à 2 dimensions, de sorte que les distances entre objets (il s'agit ici des noms d'aliments) soient proportionnelles à la similarité de ces objets. L'objectif de l'analyse est de détecter des dimensions sous-jacentes ayant une signification et permettant d'expliquer les similarités ou dissimilarités observées (distances) entre les objets étudiés.

Concernant les verbalisations: Les verbalisations de chaque sujet sont entièrement notées puis codées par deux expérimentateurs. Un tableau de " $x$ " colonnes et " $x$ " lignes est construit pour chaque groupe de participantes (témoins, anorexiques, anorexiques-boulimiques et boulimiques). Dans ce tableau en colonnes on trouve les 27 noms d'aliments et en lignes les propriétés verbalisées (gras, naturel, etc.). Le codage vise à affecter à l'intersection de chaque ligne et de chaque colonne une fréquence d'occurrence : chaque fois qu'une propriété est évoquée pour qualifier un d'aliment, la fréquence d'occurrence de cette propriété pour l'aliment donné est noté. Enfin nous avons regrouper les différentes propriétés verbalisées en fonction des trois groupes de propriétés que nous

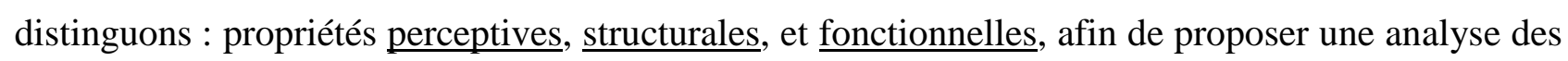
verbalisations rendant compte de la distribution des propriétés aux différents groupes d'aliments.

\subsubsection{Résultats}

Concernant la tâche de catégorisation : Dix des 27 aliments ont été catégorisés différemment par les témoins et les sujets atteints de troubles des conduites alimentaires. Il s'agit des aliments suivants :

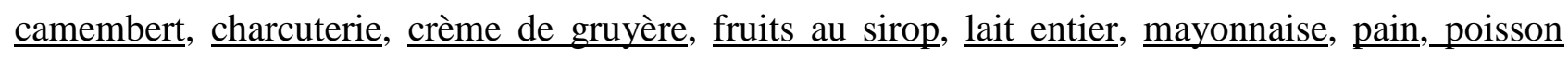
$\underline{\text { frais, }}$ pomme de terre et yaourt nature. La classification des noms d'aliments par les boulimiques est proche de celle des témoins, celle des anorexiques proche de celle des anorexiques-boulimiques.

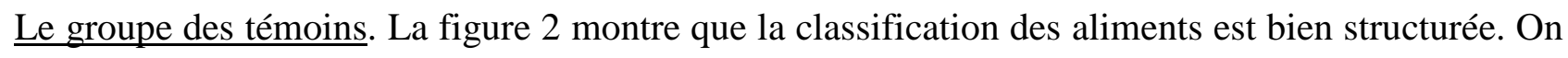
trouve en haut à gauche la catégorie que l'on peut appeler "les légumes et féculents ", catégorie composée de la tomate, des petits pois, de la macédoine de légumes, des endives cuites, des betteraves et des pommes de terre. En bas à gauche, on trouve la catégorie des "viandes et poissons" qui regroupe le poisson frais, le bœuf, l'œuf, la volaille et la charcuterie. Au centre 
gauche de cette carte, on trouve la catégorie des "aliments de complément" composée du pain et de la mayonnaise. En haut à droite, la catégorie des " fruits frais ou dérivés " qui regroupe la poire, la pomme, la banane et le jus de fruits. Un peu plus bas, toujours à droite, on trouve la catégorie des "desserts transformés" : crème dessert, gâteau crème, chocolat, gâteau sec, tarte aux fruits et fruits au sirop. Les fruits au sirop sont aussi proches des "fruits frais et dérivés”. Enfin, au bas de cette figure vers le centre - droit se trouve la catégorie des " produits laitiers" : yaourt nature, lait entier, crème de gruyère et camembert.

Insérer figure 2 ici

Le groupe des anorexiques. On observe sur la figure 3 que la catégorie des légumes et féculents, au milieu - gauche de la carte, diffère de celle des témoins par l'absence des pommes de terre. Elles sont classées dans une catégorie que l'on trouve en bas au centre du graphe, catégorie qui regroupe la mayonnaise, la charcuterie et les pommes de terre. Le pain n'est pas rangé dans une catégorie “aliments de complément" comme c'est le cas pour les témoins, mais il est proche de la catégorie des fruits. Les fruits au sirop sont proches des gâteaux (bas-droite de la carte). Enfin, la catégorisation des produits laitiers est surprenante : la crème de gruyère semble inclassable, elle est au centre en haut de la carte, le lait entier proche du gâteau à la crème (bas de la carte).

\section{Insérer figure 3 ici}

Le groupe des anorexiques-boulimiques. La figure 4 montre que la catégorie des légumes et féculents regroupe les mêmes aliments que celle des témoins à une exception : le poisson frais apparaît comme proche de cette catégorie et notamment de la tomate (droite de la carte). Dans la catégorie des viandes et poissons, la charcuterie est absente, contrairement aux témoins qui considèrent la charcuterie comme appartenant à cette catégorie. On trouve la charcuterie près du

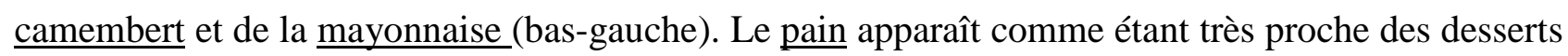
transformés (gauche de la carte). La catégorie des laitages est totalement déstructurée. Alors que, chez les témoins, la crème de gruyère, le camembert, le lait entier, le yaourt nature apparaissaient comme proches, pour les anorexiques-boulimiques, le lait entier est proche du chocolat (gauche de la carte), le camembert de la mayonnaise et de la charcuterie. Le yaourt nature est classé avec les fruits frais et dérivés (haut à droite), la crème de gruyère ne semble proche d'aucun autre aliment (haut-centre de la carte).Enfin les fruits au sirop se trouvent proches de la banane, mais plus proches des desserts transformés que des fruits frais. 
Le groupe des boulimiques. On observe sur la figure 5 que les boulimiques semblent classer les aliments de façon relativement semblable aux témoins, si ce n'est que le pain (en bas à droite) est proche de la catégorie des légumes et féculents mais plus proche des féculents que des légumes. La

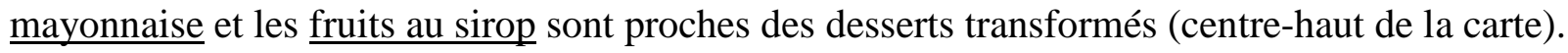

\section{Insérer figure 5 ici}

Ainsi, en ce qui concerne les témoins et les boulimiques, seuls 3 aliments (les fruits au sirop, le pain et la mayonnaise) sont rangés dans des catégories différentes. Quant aux anorexiques, ils ont catégorisé 7 aliments différemment des témoins (charcuterie, crème de gruyère, fruits au sirop, lait entier, mayonnaise, pain, et pomme de terre). Les anorexiques-boulimiques ont catégorisé 6 des 7 aliments de manière non identique aux témoins (tous sauf la pomme de terre catégorisée comme les témoins) ainsi que 3 autres aliments : le camembert, le poisson frais et le yaourt. La catégorisation des noms d'aliments par les quatre groupes de participantes (les anorexiques, les anorexiquesboulimiques, les boulimiques et les témoins) montre donc des différences notables. L'examen des critères de catégorisation avancés lors des verbalisations nous permet de mieux comprendre ces différences.

Concernant les verbalisations : L'analyse des données montre que le critère principal du groupe des témoins semble être la succession des plats. Les participantes regroupent dans une même catégorie les aliments qui constituent le plat principal, (bœuf, œuf, poisson, etc.), puis les légumes qui l'accompagnent ou qui le précèdent, les fromages ou laitages et enfin les desserts; les aliments dit de complètement (pain, mayonnaise) appartiennent à la même catégorie. Il s'agit aussi de critères nutritionnels (on trouve des protéines dans viande et le poisson, les légumes apportent l'eau, les $\underline{\text { fibres, }}$ les produits laitiers les protéines et le calcium) ou encore de critères biologiques (les fruits sont classés ensemble, les légumes, etc.). Ces critères de catégorisation font intervenir les propriétés structurales (qui décrivent de quoi l'objet est composé) et les propriétés fonctionnelles “académiques", celles qui décrivent l'utilisation des aliments "comme dans les manuels de cuisine, ou de diététique".

Les critères de catégorisation évoqués par les patientes anorexiques sont très différents de ceux des témoins : les aliments difficiles à éliminer, $\underline{\text { riches }}$ ou trop gras et de ce fait indigestes, sont considérés comme similaires. C'est le cas de la charcuterie, de la pomme de terre, de la mayonnaise 
ou encore des desserts transformés, des fruits au sirop et du lait entier. Un second critère de catégorisation fait appel à la notion de naturel : certains aliments ne sont pas naturels, "ils sont malsains car fabriqués par l'homme et sont donc mauvais pour le corps ", c'est le cas par exemple de la crème de gruyère, définie comme étant "la poubelle des fromages". Un troisième type de critère est relatif à la notion d'aliments familiers, la volaille, l'œuf sont "proche de nous", les participantes attribuent des sentiments, un état âme au poulet, par exemple, et prennent en compte ce critère de catégorisation pour regrouper le poisson, le bœuf, l'œuf et la volaille. Le pain est proche de la banane car ces aliments sont bourratifs. Nous voyons ici nettement le poids des propriétés fonctionnelles dans la catégorisation des noms d'aliments : certains aliments sont indigestes, difficiles à éliminer, donnent des aigreurs ou des renvois, ne sont pas naturels, sont trop "proche de nous" pour être consommés.

Les critères de catégorisation des anorexiques-boulimiques semblent particulièrement liés à la prise

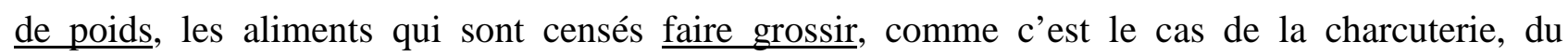
camembert et de la mayonnaise qui "contiennent plus de $45 \%$ de matière grasse" sont considérés comme proches. De même les aliments dit "gras" sont considérés comme similaires : le lait entier et les desserts transformés. De même les aliments consommés lors de crise de boulimie, car " $\underline{\text { ils calent }}$ dans le ventre", comme c'est le cas du pain et des gâteaux qui sont catégorisés ensemble. Au

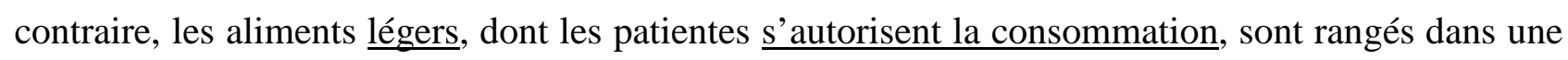
même catégorie. C'est le cas du poisson frais et la tomate pour lesquels les anorexiquesboulimiques déclarent : “c'est de l'eau, c'est léger, c'est comme si on avait rien mangé”. Le yaourt nature est proche des fruits et jus de fruits car il "n' enlève pas la faim", la banane et les fruits au sirop car ils sont "inutiles pour le corps". La crème de gruyère est considérée comme "un aliment pour enfant".

Les critères de catégorisation évoqués par les patientes boulimiques prennent clairement en compte les propriétés fonctionnelles des aliments. Il s'agit de critères du type : “cela cale, cela calme une crise de boulimie, cela permet d'éviter des aigreurs si l'on a trop mangé, cela fait grossir, cela donne des boutons, permet de lutter contre la constipation, etc.". On peut constater que les critères de catégorisation des boulimiques semblent liés uniquement à l'effet imaginé ou réel de ces aliments sur le corps. Ainsi, la mayonnaise et les fruits au sirop sont rangés dans la même catégorie que la crème dessert, le gâteau à la crème car ce sont des aliments qui donnent des boutons. De même, le yaourt et le lait entier d'une part (utilisés comme post-vomitifs après une crise de boulimie), le pain, les pommes de terre et la macédoine de légumes d'autre part sont rangés dans une même catégorie, les premiers parce qu'ils permettent d'éviter les aigreurs, les seconds parce qu' “ ils calent". 


\section{Conclusion}

Cette étude qui vise une meilleure compréhension du fonctionnement cognitif des sujets présentant des troubles des conduites alimentaires de type anorexique ou boulimique apporte des éléments nouveaux. Elle montre qu'un processus cognitif aussi élémentaire que la catégorisation semble modifié chez les patients atteints de troubles des conduites alimentaires.

Notre première étude naturaliste dans laquelle les sujets sont invités à associer en temps limité, à la lecture d'un item alimentaires toutes les représentations qui leur passent montre des différences notables entre nos quatre groupes expérimentaux. Ainsi les anorexiques et anorexiques-boulimiques produisent des représentations fortement négatives à partir des aliments alors que ces représentations sont plutôt positives pour les sujets témoins. En revanche, les boulimiques produisent des représentations dont les valences positives et négatives s'équilibrent. Ces cognitions négatives concernent principalement la viande pour les témoins, et les gâteaux pour les sujets atteints de troubles des conduites alimentaires. D’une manière générale, les témoins produisent principalement des cognitions relatives à l'hédonisme, à l'effet sur la santé et à la flaveur des aliments. Les anorexiques et anorexiques-boulimiques évoquent principalement la teneur en gras et en sucre des aliments, bien que les cognitions des anorexiques soient liées aussi à leur caractère naturel ou fabriqué et à leurs effets sur la digestion et celle des anorexiques-boulimiques à leur effets sur le corps et sur la prise de poids. Enfin les boulimiques évoquent des cognitions relatives à l'effet sur la santé et sur le transit intestinal des aliments et dans une moindre mesure des cognitions liées aux aliments consommés lors de crise ou à l'hédonisme que procure l'acte alimentaire.

Notre seconde expérimentation dans laquelle les sujets sont invités catégoriser des noms d'aliments en fonction de leurs similarités et dissemblances puis à verbaliser les raisons des regroupements évoqués permet d'affiner les premiers résultats issus de l'étude naturaliste. Ainsi 10 des 27 aliments proposés ont été catégorisés différemment par les témoins et les sujets atteints de troubles des conduites alimentaires. En ce qui concerne les témoins et les boulimiques, seuls 3 aliments sont rangés dans des catégories différentes, 7 pour les anorexiques et 9 pour les anorexiquesboulimiques.

Les différences observées entre les témoins et les sujets atteints de troubles des conduites alimentaires dans la manière dont sont catégorisés les aliments semble refléter des différences dans l'attribution singulière de propriétés fonctionnelles, liées à un traitement descendant, guidé par les théories et croyances. Ainsi, les patients anorexiques, anorexiques-boulimiques et boulimiques attribuent une importance supérieure aux propriétés fonctionnelles des aliments, comparativement aux témoins pour qui les propriétés structurales semblent primordiales. Ainsi, la catégorisation alimentaire des patients anorexiques se construit autour des conséquences de l'ingestion tant en 
termes de digestion (les aliments gras sont indigestes) que de rapport à la nature (certains aliments non naturels ou sucrés sont mauvais). La catégorisation des anorexiques-boulimique se construit autours de la prise de poids, et on peut penser que c'est pour cette raison que le sucre et le gras évoquent des cognitions négatives chez ces patients alors que chez les anorexiques le sucre et le gras semblent liés à des effets ingestifs et digestifs. Enfin les anorexiques-boulimiques et boulimiques utilisent des critères liés à la symptomatologie, certains aliments «calent «d'autres non. Chez les boulimiques l'effet des aliments sur la santé et sur le transit intestinal semble important.

Ainsi, contrairement au groupe des témoins, les patients ne catégorisent pas en fonction des usages sociaux, mais plutôt en fonction de leur relation au monde, d'investissements personnels, de leur expérience individuelle et de leur rapport à la pathologie. Chez les sujets atteints de troubles des conduites alimentaires, certaines propriétés fonctionnelles deviennent saillantes, les traitements descendants ayant un rôle prépondérant dans les traitements cognitifs. Tous les individus ont des croyances qui influencent la perception des aliments. Ainsi, chaque culture, chaque groupe humain possède une cuisine propre, c'est-à-dire une somme de représentations, de croyances et de pratiques partagées par les individus d'un même groupe, qui déterminent des classifications et un ensemble de règles portant sur la préparation, la combinaison des aliments, sur leur collecte et leur consommation (17). Ainsi, sont définies par exemple les limites du "dégoûtant", le dégoût intervenant quand la nourriture n'est pas “ bonne à penser" (23). Il s'agit alors d'un rejet purement cognitif ou idéel, pour lequel la seule pensée d'incorporer la substance provoque la révulsion, cela parce qu'on a des croyances concernant sa nature, son origine et ses qualités pour le corps. Ainsi, pour les patients atteints de troubles des conduites alimentaires, les aliments sont des Pharmakon, parfois perçus comme bons pour leur corps, mais aussi perçus comme des poisons qui peuvent détruire, faire souffrir et être source de dangers. Le traitement de l'information du stimulus alimentaire serait donc bien radicalement différent.

Les perspectives de nos recherches sont très intéressantes sur le plan fondamental comme sur le plan appliqué. Elles devraient constituer une contribution significative dans la connaissance des processus de catégorisation comme dans celui des représentations. Nous devons également retirer des informations précieuses sur le mode d'installation de croyances, et si possible sur la manière dont ces croyances peuvent devenir rigides et générer des perturbations du genre de celles que nous observons dans le dysfonctionnement des conduites alimentaires. Il apparaît nécessaire de prendre en compte ces dysfonctionnements dans les programmes thérapeutiques. 


\section{Références.}

1. BARTHELEMY JP, GUENOCHE A. Les arbres et les représentations des proximités, Paris : Masson 1988.

2. BEN-TOVIM DF, WALKER MK, FOK D et al. An adaptative of the Stroop test for measuring shape and food concerns in eating disorders: a quantitative measure of psychopathology ? International Journal of Eating Disorders 1989; 8: 681-687.

3. BEN-TOVIM DF, WALKER MK. Further evidence for the Stroop test as a quantitative measure of psychopathology in eating disorders. International Journal of Eating Disorders 1991; 10: 609-614.

4. BOON B, STROEBE W, SCHUT H, JANSEN A. Food for thought: Cognitive regulation of food intake. British Journal of Health Psychology 1998, 3, 27

5. BRUCH H. Psychotherapy in anorexia nervosa. International Journal of Eating Disorders 1982; 1(4): 3-14.

6. CALLANAN MA. Development of Object Categories and Inclusion Relations: Preschoolers' Hypotheses About Word. In D. Luce, \& P. Suppes (Eds.). Contemporary developments in mathematical psychology. San Francisco: Freeman 1989; 2: 57-105.

7. COOPER M J, ANASTASIADES P, FAIRBURN, CG. Selective processing of eating, shape and weight related words in persons with bulimia nervosa. Journal of Abnormal Psychology 1992, 101(2), 352-355.

8. COOPER MJ, CLARK DM, FAIRBURN CG. An experimental study of the relationship between thoughts and eating behaviour in bulimia nervosa. Behaviour Research and Therapy 1993; 31(8): 749-757.

9. COOPER MJ, FAIRBURN CG. Thoughts about eating weight and shape in anorexia nervosa and bulimia nervosa. Behaviour Research and Therapy 1992; 30: 501-511.

10. COOPER MJ, TODD G, WELLS A. Content, origins, and consequences of dysfunctional beliefs in anorexia nervosa and bulimia nervosa. Journal of Cognitive Psychotherapy 1998, 12 (3), 213-230

11. CORDIER F, TIJUS C. Object properties: A typology. Cahiers de Psychologie Cognitive/Current Psychology of Cognition 2001; 20(6): 445-472.

12. DRITSCHEL BH, WILLIAMS K, COOPER PJ. Cognitive distortions among women experiencing bulimic episodes. International Journal of Eating Disorders 1991; 10 (5): 547-555.

13. FAIRBURN C, SHAFRAN GR, COOPER Z. A cognitive behavioural theory of anorexia nervosa, Behavior Research and Therapy 1999; 37(1): 1-13. 
14. FAIRBURN CG, BEGLIN S.J. Assessment of eating disorders: interview or self-report questionnaire? International Journal of Eating Disorders 1994; 16 (4): 363-370.

15. FAIRBURN CG, COOPER PKJ, COOPER MJ, Mc KENNA FP, ANASTASIADES P Selective information processing in bulimia nervosa. International Journal of Eating Disorders 1991; 10: 415-422.

16. FAIRBURN CG, STEERE J, COOPER PJ. Assessment of the specific psychopathology. In M.M. Fichter (Ed.). Bulimia Nervosa: Basic research, diagnosis and therapy, New-York: Wiley 1990; 37-56.

17. FISCHLER C. L'homnivore. Paris : Odile Jacob 2001.

18. GARNER DM, OLMSTED MP, POLIVY J. Development and validation of a multidimensional eating inventory for anorexia nervosa and bulimia. International Journal of Eating Disorders 1983; 2 (2): 15-23.

19. GARNER PM, BEMIS KM. A cognitive behavioral approach to anorexia nervosa, Cognitive Therapy and Research 1982; 6: 123-150.

20. HARRIS EC, BARRACLOUGH B. Excess mortality of mental disorder. British Journal Psychiatry, 1998; 173: 11-53.

21. KOMATSU LK. Recent views of conceptual structure. Psychological Bulletin 1992, 112, 500526.

22. LEUNG N., WALLER G, THOMAS G. Core beliefs in anorexic and bulimic women. Journal of Nervous and Mental Disease 1999, 187(12), 736-741.

23. LEVI-STRAUSS C. Le totémisme aujourd'hui. Paris : P.U.F. 1962

24. MALT B. Category coherence in cross-cultural perspective. Cognitive Psychology 1995; 29 : 85-148.

25. MARKMAN AB, MAKIN D. Referential communication and category acquisition, Journal of Experimental Psychology 1998; 127 (4): 331-354.

26. MIRABEL-SARRON C, BICHINDARITZ I, SAMUEL-LAJEUNESSE B. The food cognitions questionnaire: preliminary study. World Congress of Psychiatry: Madrid 1996.

27. MIRABEL-SARRON C, FOULON C, SAMUEL-LAJEUNESSE B. Essai d'approche des troubles de la représentation mentale à partir de stimulus visuels chez des patients anorexiques. L'encéphale 1992, 18, 623-629

28. MIRABEL-SARRON C, SAMUEL-LAJEUNESSE B. Assessment of food cognition. Congres of Food Choice, Bruxelles Juillet 1992.

29. MIRABEL-SARRON C, SAMUEL-LAJEUNESSE B. Evaluation des dysfonctionnements cognitifs dans les troubles des conduites alimentaires, L’encéphale 1998 ; Versailles 5-6 juin 1997 : 56-59. 
30. MIZES JS. Criterion-related validity of the anorectic cognitions questionnaire. Additive Behaviours 1990; $15: 153-163$.

31. MURPHY GL, MEDIN DL. The role of theories in conceptual coherence. Psychological Review, 92 1985, 289-316.

32. NELSON K. Le développement de la représentation sémantique chez l'enfant. Psychologie Française 1985; 30: 261-268.

33. PHELAN P.W. Cognitive correlates of bulimia: the bulimic thoughts questionnaire International Journal of Eating Disorders 1987; 6 (5): 593-607

34. ROSCH E. Cognitive representations of semantic categories. Journal of Experimental Psychology: Human Perception and Performance 1975; 1: 303-322.

35. ROSS BH, \& MURPHY GL. Food for thought: cross-classification and category organization in a complex real-world domain. Cognitive Psychology 1999, 38, 495-555.

36. SAMUEL-LAJEUNESSE B, FOULON C. Les troubles des conduites alimentaires. Masson 1994.

37. SCHULMAN RG, KINDER BN, POWERS PS, PRANGE M, GLEGHORN A. The development of a scale to measure cognitive distortions in bulimia. Journal of Personality Assessment 1986; 50: 630-639.

38. THOMPSON DA, BERG KM, SHATFORD LA. The heterogeneity of bulimic symptomatology: cognitive and behavioral dimensions, International Journal of EatingDisorders 1987; 6 (2): 215-234

39. TVERSKY B, HEMENWAY K. Objects, Parts, and Categories, Journal of Experimental Psychology General 1984; 113: 172-191.

40. URDAPILLETA I, BERNARD JM. Quasi-implication in the attribution of verbal descriptors of sensory attributes. Current psychology letters : behaviour, brain and cognition $2002 ; 2002 / 3$, $\mathrm{N}^{\circ} 9$

41. URDAPILLETA I, NICKLAUS S, TIJUS CA. Sensory evaluation based on verbal judments. Journal of Sensory Studies, 14 (1): 79-96.

42. VON HIPPEL W, HAWKINS C, NARAYAN S. Personality and perceptual expertise: Individual differences in perceptual identification. Psychological Science 1994, 5, 401-406

43. VRIGNAUD P. Approche différentielle de la typicalité. Thèse de Doctorat de l'Université Paris 51998.

44. WHITE CW, MONTGOMERY DA. Memory colors in afterimages: A bicentennial demonstration. Perception \& Psychophysics 1976, 19, 371-374

45. WHORF BJ. Languages and logic. in J. B. Carroll (ed.) Language, Thought, and Reality: Selected papers of Benjamin Lee Whorf. MIT Press (1956). Cambridge, Mass. 1941, (pp. 233245). 
Légende des figures

Figure 1. Répartition des cognitions négatives selon les types de cognitions alimentaires pour chaque groupe de participantes.

Figure 2. Analyse des proximités relatives au groupe des témoins

Figure 3. Analyse des proximités relatives au groupe des anorexiques

Figure 4. Analyse des proximités relatives au groupe des anorexiques-boulimiques

Figure 5. Analyse des proximités relatives au groupe des boulimiques 


\section{Témoins}

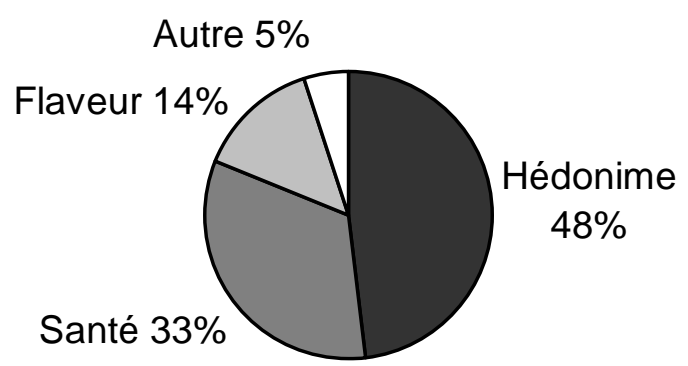

\section{Anorexiques}

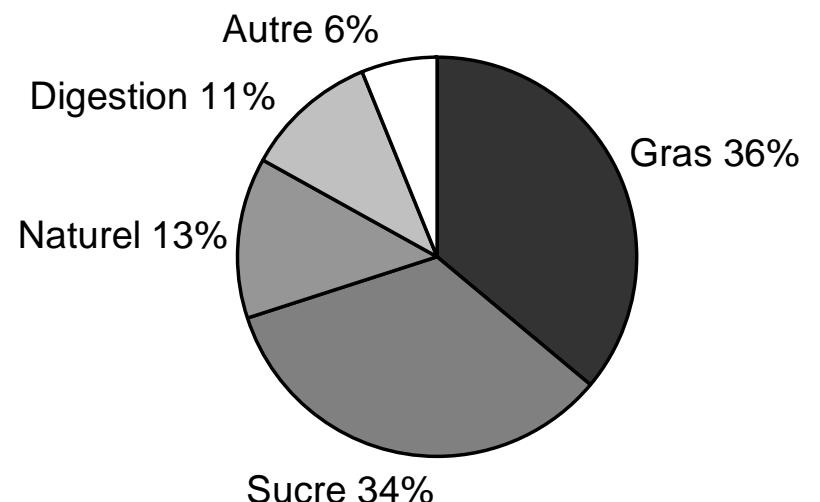

\section{Anorexiques-boulimiques}

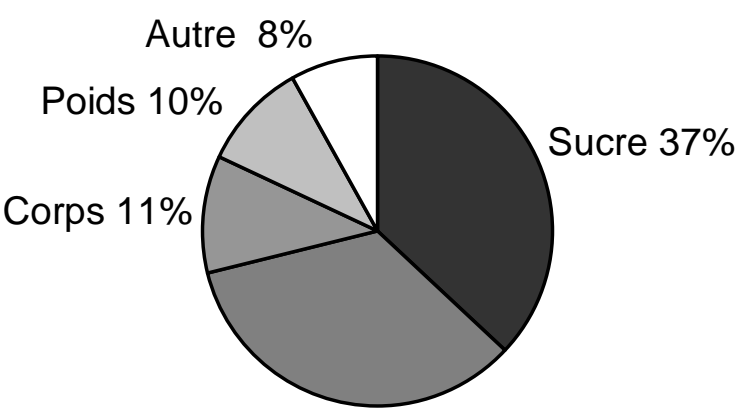

Gras 34\% 


\section{Boulimiques}

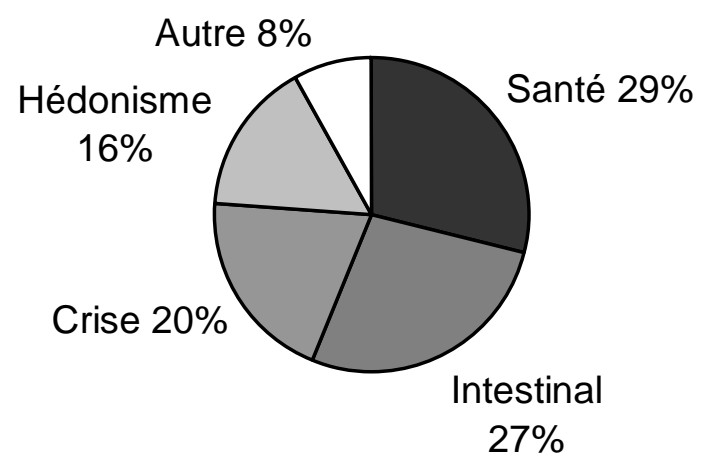




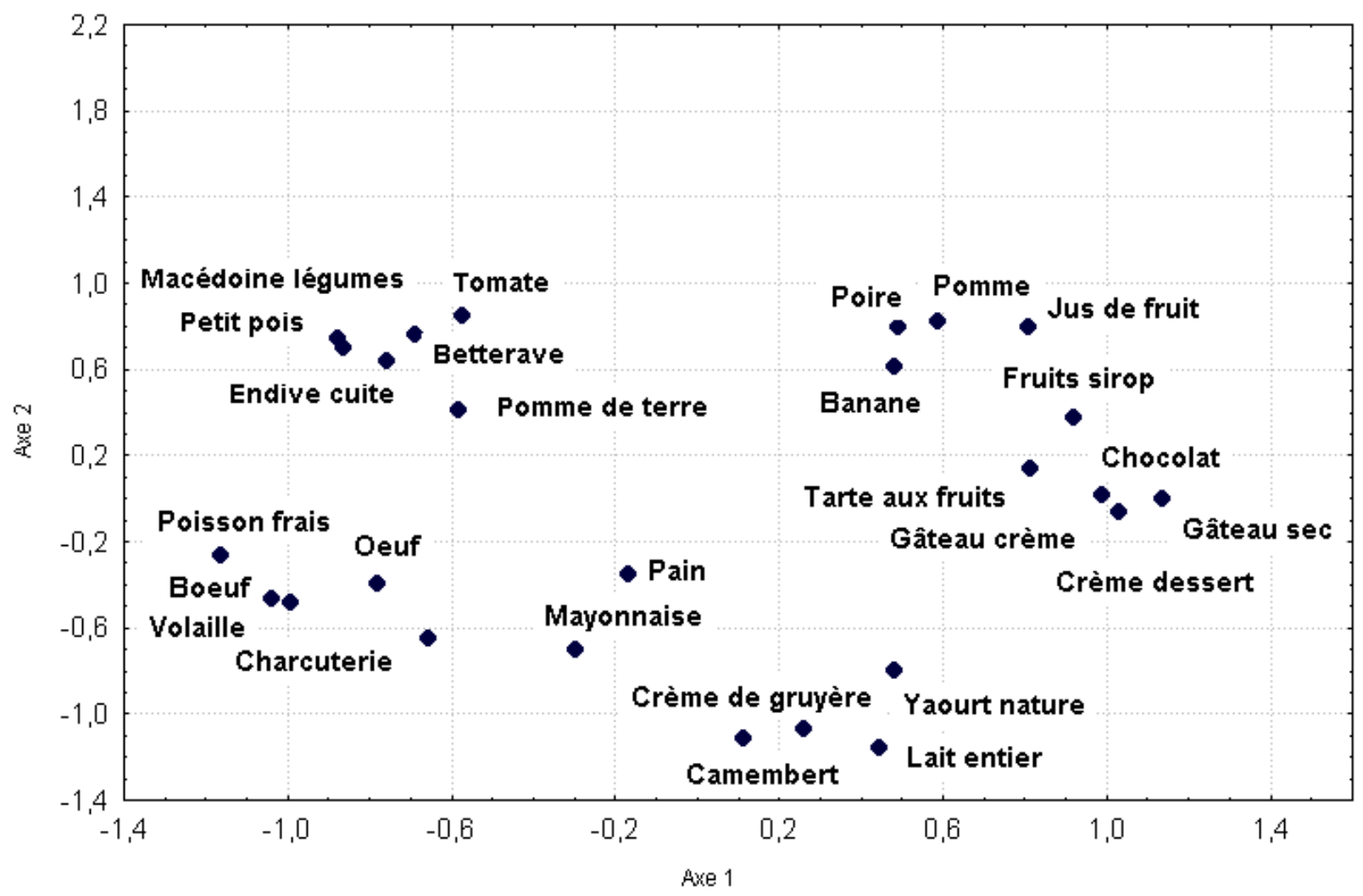




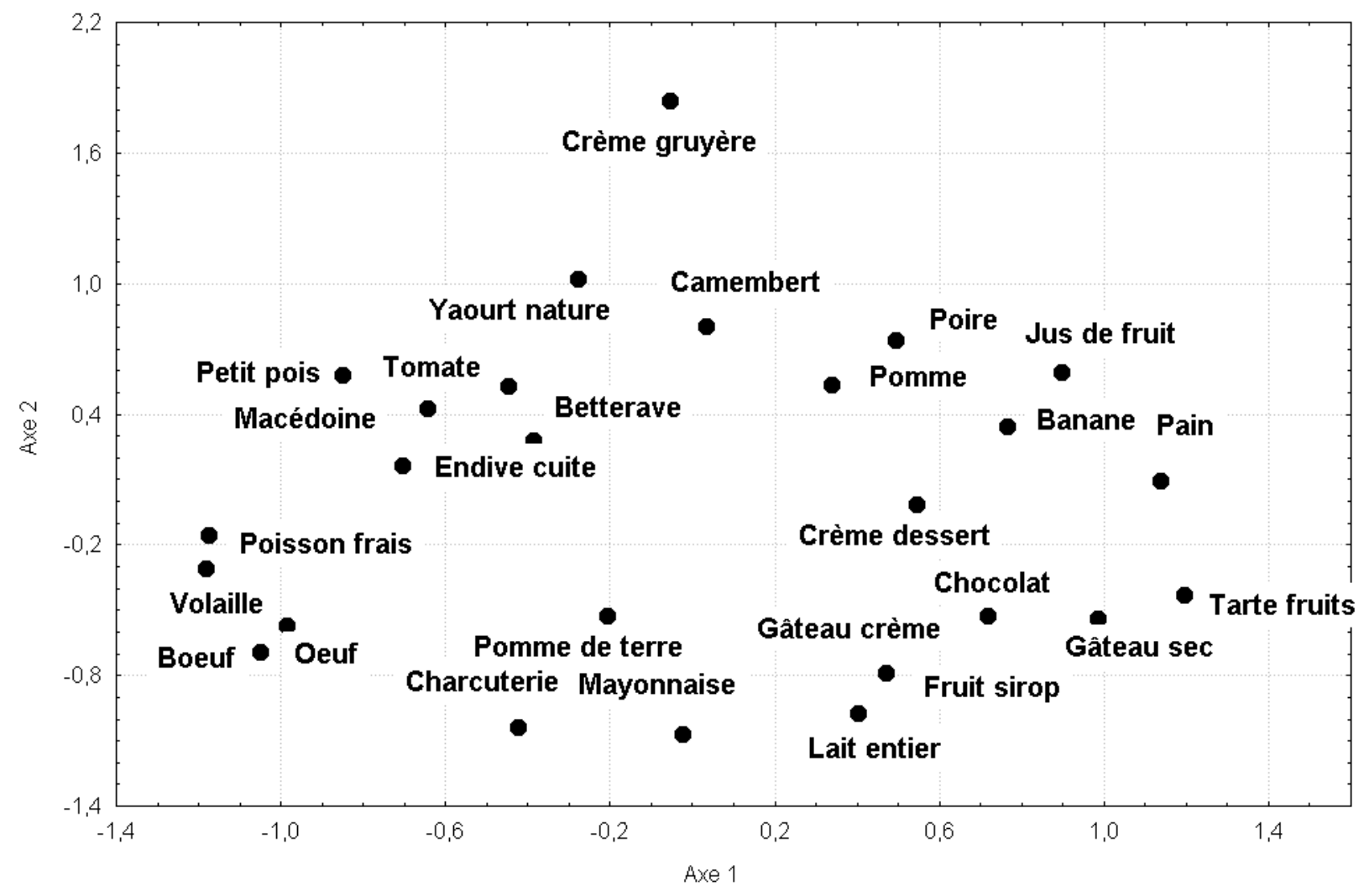



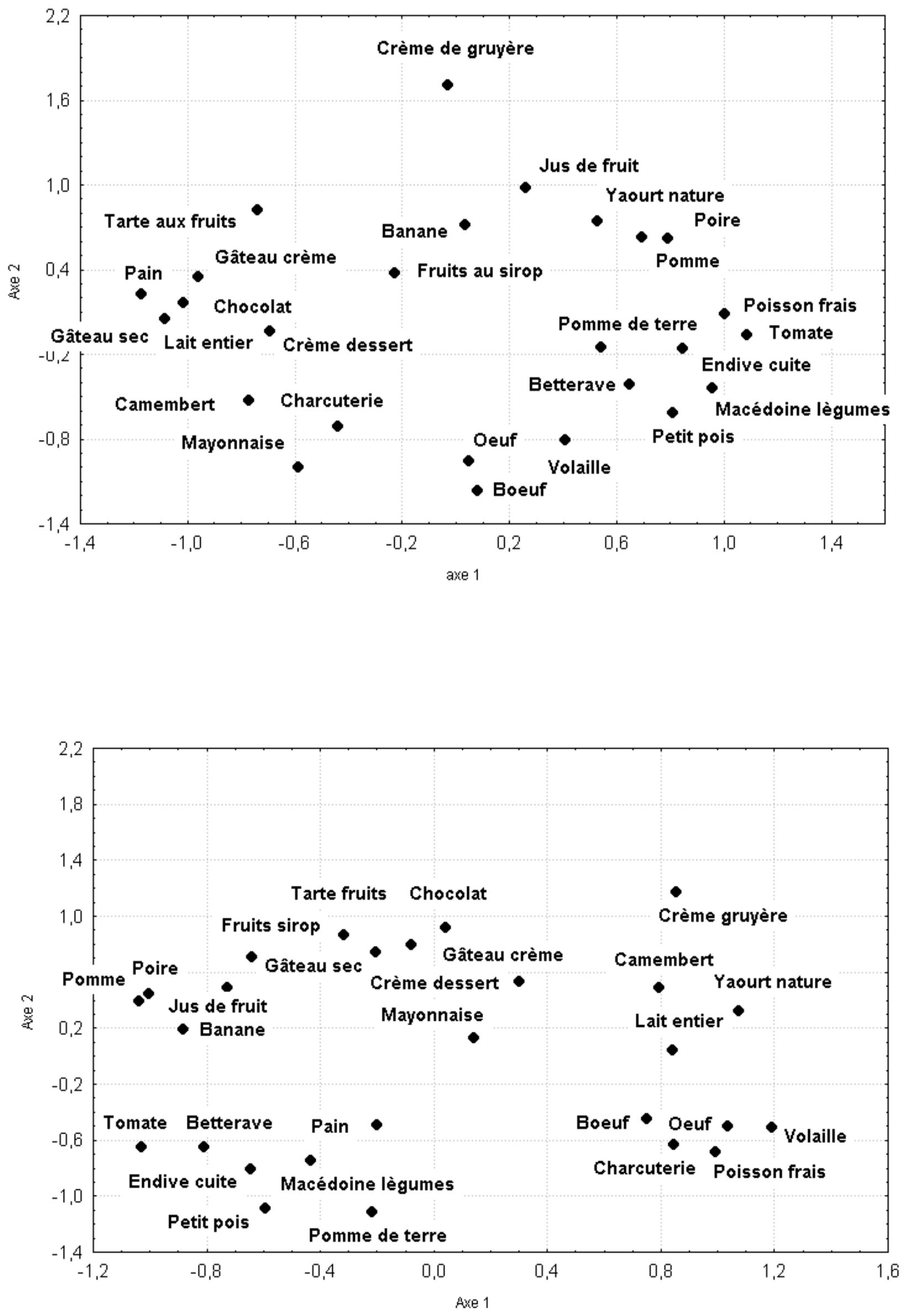